Article

\title{
On the Domain of the Fibonacci Difference Matrix
}

\author{
Fevzi Yaşar ${ }^{1, *(1)}$ and Kuddusi Kayaduman ${ }^{2}$ \\ 1 Kilis Yatırım Destek Ofisi, Şehitler Mah. Cambazlar Sok. No:9, Kilis 79000, Turkey \\ 2 Faculty of Arts and Sciences, Department of Mathematics, Gaziantep University, Gaziantep 27310, Turkey; \\ kayaduman@gantep.edu.tr \\ * Correspondence: fevziyasar@hotmail.com
}

Received: 31 December 2018; Accepted: 19 February 2019; Published: 21 February 2019

check for updates

\begin{abstract}
Matrix $\hat{F}$ derived from the Fibonacci sequence was first introduced by Kara (2013) and the spaces $l_{p}(F)$ and $l_{\infty}(F) ;(1 \leq p<\infty)$ were examined. Then, Başarır et al. (2015) defined the spaces $c_{0}(F)$ and $c(F)$ and Candan (2015) examined the spaces $c(F(r, s))$ and $c_{0}(F(r, s))$. Later, Yaşar and Kayaduman (2018) defined and studied the spaces $c s(F(s, r))$ and $b s(F(s, r))$. In this study, we built the spaces $c s(F)$ and $b s(F)$. They are the domain of the matrix $F$ on $c s$ and $b s$, where $F$ is a triangular matrix defined by Fibonacci Numbers. Some topological and algebraic properties, isomorphism, inclusion relations and norms, which are defined over them are examined. It is proven that $c s(F)$ and $b s(F)$ are Banach spaces. It is determined that they have the $\gamma, \beta, \alpha$-duals. In addition, the Schauder base of the space $\operatorname{cs}(F)$ are calculated. Finally, a number of matrix transformations of these spaces are found.
\end{abstract}

Keywords: matrix transformations; Fibonacci numbers; sequence spaces; Fibonacci double band matrix; $\gamma, \beta, \alpha$-duals

\section{Introduction}

Cooke [1] formulated the theory of infinite matrices in the book "Infinite Matrices and Sequence Spaces". Many researchers have investigated infinite matrices after the publication of this book in 1950. In most of these studies, the domain of infinite matrices on a sequence space was studied. In this study, we address the question: What are the properties of the domain of the Fibonacci band matrix on sequence spaces $b s$ and $c s$ ? The domain of the Fibonacci band matrix creates a new sequence space. We handle algebraic properties of this new space in order to determine its duals and its place among other known spaces, and to characterize the matrix transformations of this space.

One difficulty of this study is to determine whether the new space is the contraction or the expansion, or the overlap of the original space. Another difficulty is to determine the matrix transformations on this space and into this space. For the first problem, we give a few inclusion theorems. For the second problem, we use the matrix transformation between the standard sequence spaces and two theorems.

Generating a new sequence space and researching on its properties have been important in the studies on the sequence space. Some researchers examined the algebraic properties of the sequence space while others investigated its place among other known spaces and its duals, and characterized the matrix transformations on this space.

We can create a new sequence space by using the domain of infinite matrices. Ng-Lee [2] first investigated the domain of an infinite matrix in 1978. In the same period, Wang [3] created a new sequence space by using another infinite matrix. Many researchers such as Malkovsky [4], Altay, and Başar [5] followed these studies. This topic was studied intensively after 2000. 
Leonardo Fibonacci invented Fibonacci numbers. He introduced Fibonacci numbers originated from a rabbit problem. These numbers create a number sequence:

$$
1,1,2,3,5,8,13,21,34,55,89, \ldots .
$$

This sequence has important properties and applications in various fields.

Let us indicate the Fibonacci sequence by $\left(f_{n}\right) . f_{n}$ is defined as

$$
f_{n}=f_{n-1}+f_{n-2}(n \geq 2)
$$

with $f_{0}=f_{1}=1$. The golden ratio is

$$
\lim _{n \rightarrow \infty} \frac{f_{n+1}}{f_{n}}=\frac{1+\sqrt{5}}{2}=\phi .
$$

Let us indicate the set of all real-valued sequences with $w$ and list some subspaces of $w$ called standard sequence spaces.

$$
\begin{gathered}
c=\left\{r=\left(r_{k}\right) \in w: \lim _{k \rightarrow \infty}\left|r_{k}-p\right|=0 \text { for some } p \in \mathbb{C}\right\}, \\
c_{0}=\left\{r=\left(r_{k}\right) \in w: \lim _{k \rightarrow \infty}\left|r_{k}\right|=0\right\}, \\
b s=\left\{r=\left(r_{k}\right) \in w: \sup _{n \in \mathbb{N}}\left|\sum_{k=0}^{n} r_{k}\right|<\infty\right\}, \\
c s_{0}=\left\{r=\left(r_{k}\right) \in w: \lim _{n \rightarrow \infty}\left|\sum_{k=0}^{n} r_{k}\right|=0\right\}, \\
l_{\infty}=\left\{r=\left(r_{k}\right) \in w: \sup _{k \in \mathbb{N}}\left|r_{k}\right|<\infty\right\}, \\
b v=\left\{r=\left(r_{k}\right) \in w: \sum_{k=0}^{\infty}\left|r_{k}-r_{k-1}\right|<\infty\right\}, \\
\quad b v_{0}=b v \cap c_{0} \\
l_{p}=\left\{r=\left(r_{k}\right) \in w: \sum_{k=0}^{\infty}\left|r_{k}\right|^{p}<\infty, 0<p<\infty\right\}, \\
l_{1}=\left\{r=\left(r_{k}\right) \in w: \sum_{k=0}^{\infty}\left|r_{k}\right|<\infty\right\},
\end{gathered}
$$

Now let us take real valued infinite matrix $T=\left(t_{n k}\right)$, where $t_{n k}$ is a real number for every $n, k \in \mathbb{N}$ Let $A$ and $B$ be sequence spaces. Sequence $T x=\left\{T_{n}(x)\right\}$ is T-transform of $a$ for every $a=\left(a_{k}\right) \in A$. Here, $T a \in B$ and

$$
T_{n}(a)=\sum_{k} t_{n k} a_{k}
$$

and $T_{n}(a) \rightarrow t$ ( $t$ exists for every $\left.n \in \mathbb{N}\right)$. Then, $T$ is called a matrix transformation from $A$ to $B$.

Now let us take infinite matrix $T$ and sequence space $\delta$ to define domain of infinite matrix $T$. The domain of the matrix $T$ on $\delta$ is characterized by

$$
\delta_{T}=\left\{x=\left(x_{k}\right) \in w: T x \in \delta\right\} .
$$


Many reserachers have studied the domain of a matrix on a sequence space. For more detailed information on these new sequence spaces, see references [6-26].

The Fibonacci difference matrix $F$ was first introduced by Kara [27] in 2013. $F$ is derived from $\left(f_{n}\right)$. In this study, Kara [27] defined the spaces $l_{p}(F)$ and $l_{\infty}(F) ;(1 \leq p<\infty)$. After this study the $c_{0}(F(r, s))$ and $c(F(r, s))$ was introduced by Candan [28], in 2015, where $F(r, s)$ is a generalized Fibonacci matrix. Candan and Kara [19] introduced and examined $l_{p}(F(r, s)) ;(1 \leq p<\infty)$. In 2018, Yaşar and Kayaduman [29] defined and examined $c s(F(s, r))$ and $b s(F(s, r))$ and Kayaduman and Yaşar [30] studied spaces $b s\left(N^{t}\right)$ and $\operatorname{cs}\left(N^{t}\right)$, where $N^{t}$ is a Nörlund matrix.

Let $\delta$ be a sequence space. The $\gamma, \beta, \alpha$-duals of $\delta$ are defined, respectively, as follows

$$
\begin{aligned}
& \delta^{\gamma}=\left\{x=\left(x_{k}\right) \in w: x s=\left(x_{k} s_{k}\right) \in b s \text { for all } s \in \delta\right\}, \\
& \delta^{\beta}=\left\{x=\left(x_{k}\right) \in w: x s=\left(x_{k} s_{k}\right) \in c s \text { for all } s \in \delta\right\}, \\
& \delta^{\alpha}=\left\{x=\left(x_{k}\right) \in w: x s=\left(x_{k} s_{k}\right) \in l_{1} \text { for all } s \in \delta\right\} .
\end{aligned}
$$

In this study, spaces $c s(F)$ and $b s(F)$ are introduced and the related notations are given in Section 2. In addition, some topological and algebric properties, isomorphism, inclusion relations and norms which are defined over them are examined. The $\gamma, \beta, \alpha$-duals of these spaces are determined in Section 3. The Schauder base of space $c s(F)$ are calculated. Finally, many matrix transformations of these spaces are found. In the last section, the results and previous studies and the working hypotheses are discussed.

A detailed literature review was performed before this study was started. Scans were made on related articles, magazines, and books. As a result of these scans, the part related to our subject was synthesized and the results were noted. These results were then applied to our problem area. Finally, the results of this study were obtained.

\section{Results}

\subsection{The Domain of Fibonacci Difference Matrix F on Bounded and Convergent Series}

In this section, $c s(F)$ and $b s(F)$ are introduced. Related notations are given. In addition, some topological and algebric properties, isomorphism, inclusion relations, and norms defined over them are examined.

For similar studies, see refeerences [19] and [27-34].

Let spaces $c s(F)$ and $b s(F)$ be the domain of the matrix $F$ on $c s$ and $b s$, where $F=\left\{f_{n k}\right\}$ infinite matrix is defined by $\left(f_{n}\right)$

$$
f_{n k}=\left\{\begin{array}{cc}
-\frac{f_{n+1}}{f_{n}}, & k=n-1 \\
\frac{f_{n}}{f_{n+1}}, & k=n \\
0, & 0 \leq k<n-1 \text { or } n<k
\end{array}\right.
$$

for all $k, n \in \mathbb{N}$. Then we inroduce $c s(F)$ and $b s(F)$ as

$$
\begin{gathered}
c s(F)=\left\{x=\left(x_{k}\right) \in w:\left(\sum_{k=0}^{n}\left(\frac{f_{k}}{f_{k+1}} x_{k}-\frac{f_{k+1}}{f_{k}} x_{k-1}\right)\right)_{n} \in c\right\} \\
b s(F)=\left\{x=\left(x_{k}\right) \in w: \sup _{n \in \mathbb{N}}\left|\sum_{k=0}^{n}\left(\frac{f_{k}}{f_{k+1}} x_{k}-\frac{f_{k+1}}{f_{k}} x_{k-1}\right)\right|<\infty\right\} .
\end{gathered}
$$

We can see $c s(F)=(c s)_{F}$ and $b s(F)=(b s)_{F}$ by using Equation (2). 
Let the inverse matrix of $F$ be $F^{-1}$. For all $k, n \in \mathbb{N}, F^{-1}=\left\{F^{-1}{ }_{n k}\right\}$ is found as

$$
f_{n k}^{-1}=\left\{\begin{array}{cc}
\frac{f_{n+1}^{2}}{f_{k} f_{k+1}}, & 0 \leq k \leq n \\
0, & k>n
\end{array}\right.
$$

Let us take sequence $x=\left(x_{n}\right)$. If $y=F x$, then we calculate as

$$
y_{n}=(F x)_{n}= \begin{cases}x_{0}, & n=0 \\ \frac{f_{n}}{f_{n+1}} x_{n}-\frac{f_{n+1}}{f_{n}} x_{n-1}, & n \geq 1 .\end{cases}
$$

Herefrom, if we calculate inverse of $F$, then we find that $x=F^{-1} y$ and

$$
x_{n}=\sum_{k=0}^{n} \frac{f_{n+1}^{2}}{f_{k} f_{k+1}} y_{k}
$$

Now, let us give some theorems related to our study.

Theorem 1. bs $(F)$ is a linear space.

Proof. The proof is left to the reader since it is easy to show.

Theorem 2. cs $(F)$ is a linear space.

Proof. The proof is left to the reader since it is easy to show.

Theorem 3. $b s(F)$ is a normed space with:

$$
\|x\|=\sup _{n \in \mathbb{N}}\left|\sum_{j=0}^{n}\left(\frac{f_{k}}{f_{k+1}} x_{k}-\frac{f_{k+1}}{f_{k}} x_{k-1}\right)\right|
$$

Proof. The proof is left to the reader since it is easy to show.

Theorem 4. $c s(F)$ is a normed space with Equation (6).

Proof. The proof is left to the reader since it is easy to show.

Theorem 5. bs $(F)$ is isomorphic to bs.

Proof. Let us take $T: b s(F) \rightarrow b s$ mentioned Equation (4) by $x \rightarrow y=T x=F x$. It is easy to see that $T$ is linear and injective.

We must find $T$ is surjective. Let $y=\left(y_{n}\right) \in b s$. By using Equation (5) and Equation (6), we see

$$
\begin{aligned}
\|x\| & =\sup _{n \in \mathbb{N}}\left|\sum_{k=0}^{n}\left(\frac{f_{k}}{f_{k+1}} x_{k}-\frac{f_{k+1}}{f_{k}} x_{k-1}\right)\right| \\
& =\sup _{n \in \mathbb{N}}\left|\sum_{k=0}^{n}\left(\frac{f_{k}}{f_{k+1}}\left(\sum_{j=0}^{k} \frac{f_{k+1}^{2}}{f_{j} f_{j+1}} y_{j}\right)-\frac{f_{k+1}}{f_{k}}\left(\sum_{k=0}^{k-1} \frac{f_{k+1}^{2}}{f_{j} f_{j+1}} y_{j}\right)\right)\right| \\
& =\sup _{n \in \mathbb{N}}\left|\sum_{k=0}^{n} y_{k}\right|=\|y\|_{b s}<\infty .
\end{aligned}
$$

We see that $x \in b s(F)$. Hence, $T$ is surjective. In addition, $b s(F)$ and $b s$ izometric because $\|x\|_{b s(F)}=$ $\|y\|_{b s}$. 
Theorem 6. $\operatorname{cs}(F)$ is isomorphic to cs.

Proof. The proof can be made similar to Theorem 5, so it is left to the reader.

Theorem 7. bs $(F)$ is a Banach space with Equtaion (6).

Proof. It is easy to see the norm conditions are ensured. Let a Cauchy sequence $x^{i}=\left(x_{k}{ }^{i}\right)$ in $b s(F)$ for each $i \in \mathbb{N}$. For all $k \in \mathbb{N}$, we have

$$
y_{k}^{i}=\frac{f_{k}}{f_{k+1}} x_{k}^{i}-\frac{f_{k+1}}{f_{k}} x_{k-1}^{i}
$$

from Equation (4). For all $\varepsilon>0$ there is $n_{0}=n_{0}(\varepsilon)$ such that

$$
\begin{aligned}
\left\|x^{i}-x^{m}\right\|_{b s(F)} & =\sup _{n \in \mathbb{N}}\left|\sum_{k=0}^{n}\left(\frac{f_{k}}{f_{k+1}}\left(x_{k}^{i}-x_{k}^{m}\right)-\frac{f_{k+1}}{f_{k}}\left(x_{k-1}^{i}-x_{k-1}^{m}\right)\right)\right| \\
& =\sup _{n \in \mathbb{N}}\left|\sum_{k=0}^{n}\left(y_{k}^{i}-y_{k}^{m}\right)\right|=\left\|y^{i}-y^{m}\right\|_{b s}<\varepsilon
\end{aligned}
$$

for all $i, m \geq n_{0} . y_{i} \rightarrow y(i \rightarrow \infty)$ such that $y \in b s$ exists, since $b s$ is complete. Since $b s$ and $b s(F)$ are isomorphic, $b s(F)$ is complete. It hereby is a Banach space.

Theorem 8. $c s(F)$ is a Banach space with Equation (6).

Proof. It is easy to see the norm conditions are ensured. Let a Cauchy sequence $x^{i}=\left(x_{k}^{i}\right)$ in $\operatorname{cs}(F)$ for each $i \in \mathbb{N}$. For all $k \in \mathbb{N}$, we have

$$
y_{k}^{i}=\frac{f_{k}}{f_{k+1}} x_{k}^{i}-\frac{f_{k+1}}{f_{k}} x_{k-1}^{i}
$$

from Equation (4). For all $\varepsilon>0$, there is $n_{0}=n_{0}(\varepsilon)$ such that

$$
\begin{aligned}
\left\|x^{i}-x^{m}\right\|_{\mathcal{C S}(F)} & =\sup _{n \in \mathbb{N}}\left|\sum_{k=0}^{n}\left(\frac{f_{k}}{f_{k+1}}\left(x_{k}^{i}-x_{k}^{m}\right)-\frac{f_{k+1}}{f_{k}}\left(x_{k-1}^{i}-x_{k-1}^{m}\right)\right)\right| \\
& =\sup _{n \in \mathbb{N}}\left|\sum_{k=0}^{n}\left(y_{k}^{i}-y_{k}^{m}\right)\right|=\left\|y^{i}-y^{m}\right\|_{\mathcal{C S}}<\varepsilon
\end{aligned}
$$

for all $i, m \geq n_{0} . \quad y_{i} \rightarrow y(i \rightarrow \infty)$ such that $y \in c s$ exists, since $c s$ is complete. Since $c s$ and $\operatorname{cs}(F)$ are isomorphic, $\operatorname{cs}(F)$ is complete. It hereby is a Banach space.

Now, let $R=\left(r_{n k}\right)$ infinite matrix. Let us list the following:

$$
\begin{gathered}
\sup _{n \in \mathbb{N}} \sum_{k}\left|r_{n k}\right|<\infty, \\
\lim _{k} r_{n k}=0 \text { for each } n \in \mathbb{N}, \\
\sup _{m} \sum_{k}\left|\sum_{n=0}^{m}\left(r_{n k}-r_{n, k+1}\right)\right|<\infty, \\
\lim _{n} \sum_{k} r_{n k}=p \text { for each } \mathrm{k} \in \mathrm{N}, p \in \mathbb{C}, \\
\sup _{n} \sum_{k}\left|r_{n k}-r_{n, k+1}\right|<\infty, \\
\lim _{n} r_{n k}=a_{k} \text { for each } \mathrm{k} \in \mathbb{N}, a_{k} \in \mathbb{C},
\end{gathered}
$$




$$
\begin{gathered}
\sup _{N, K \in \mathcal{F}}\left|\sum_{n \in \mathbb{N}} \sum_{k \in \mathbb{N}}\left(r_{n k}-r_{n, k+1}\right)\right|<\infty, \\
\sup _{N, K \in \mathcal{F}}\left|\sum_{n \in \mathbb{N}} \sum_{k \in \mathbb{N}}\left(r_{n k}-r_{n, k-1}\right)\right|<\infty, \\
\lim _{n}\left(r_{n k}-r_{n, k+1}\right)=a \text { for each } \mathrm{k} \in \mathbb{N}, a \in \mathbb{C}, \\
\lim _{n} \sum_{k}\left|r_{n k}-r_{n, k+1}\right|=\sum_{k}\left|\lim \left(r_{n k}-r_{n, k+1}\right)\right|, \\
\sup _{N, K \in \mathcal{F}}\left|\sum_{n \in \mathbb{N}} \sum_{k \in \mathbb{N}}\left[\left(r_{n k}-r_{n-1, k}\right)-\left(r_{n, k-1}-r_{n-1, k-1}\right)\right]\right|<\infty . \\
\lim _{n} \sum_{k}\left|r_{n k}-r_{n, k+1}\right|=0 \text { uniformly in } n, \\
\sup _{N, K \in \mathcal{F}}\left|\sum_{n \in \mathbb{N}} \sum_{k \in \mathbb{N}}\left[\left(r_{n k}-r_{n, k+1}\right)-\left(r_{n-1, k}-r_{n-1, k+1}\right)\right]\right|<\infty, \\
\lim _{m} \sum_{k} \mid \sum_{n=0}^{m}\left(\sum_{n k}\left(r_{n k}-r_{n, k+1}\right) \mid=0,\right. \\
\left.\sup _{m \in \mathbb{N}} \mid \lim _{k} \sum_{n=0} \sum_{n, k+1}\right)\left|=\sum_{k}\right| \sum_{n}\left(r_{n k}-r_{n, k+1}\right) \mid
\end{gathered}
$$

The collection of all finite subsets of $\mathbb{N}$ denoted by $\mathcal{F}$.

Lemma 9. Let us suppose infinite matrix $R=\left(r_{n k}\right)$. Then,

(1) $R=\left(r_{n k}\right) \in\left(b s, l_{\infty}\right)$ iff Equations (11) and (8) hold [35].

(2) $R=\left(r_{n k}\right) \in(c s, c)$ iff Equations (11) and (12) hold [36].

(3) $R=\left(r_{n k}\right) \in\left(b s, l_{1}\right)$ iff Equations (13) and (8) hold [37].

(4) $R=\left(r_{n k}\right) \in\left(c s, l_{1}\right)$ iff Equation (14) holds [35].

(5) $R=\left(r_{n k}\right) \in(b s, c)$ iff Equations (8), (15) and (16) hold [37].

(6) $R=\left(r_{n k}\right) \in\left(c s, l_{\infty}\right)$ iff Equations (17) and (11) hold [35].

(7) $R=\left(r_{n k}\right) \in\left(b s, c_{0}\right)$ iff Equations (18) and (8) hold [35].

(8) $R=\left(r_{n k}\right) \in\left(b s, c s_{0}\right)$ iff Equations (19) and (8) hold [38].

(9) $R=\left(r_{n k}\right) \in(b s, c s)$ iff Equations (20) and (8) hold [38].

(10) $R=\left(r_{n k}\right) \in(b s, b v)$ iff Equations (21) and (8) hold [38].

(11) $R=\left(r_{n k}\right) \in(b s, b s)$ iff Equations (8) and (9) hold [38].

(12) $R=\left(r_{n k}\right) \in(c s, c s)$ iff Equations (9) and (10) hold [39].

(13) $R=\left(r_{n k}\right) \in\left(b s, b v_{0}\right)$ iff Equations (21), (18) and (21) hold [35].

(14) $R=\left(r_{n k}\right) \in\left(c s, c_{0}\right)$ iff Equations (11) and (12) hold with $a_{k}=0$ for all $k \in \mathbb{N}$ [40].

(15) $R=\left(r_{n k}\right) \in(c s, b s)$ iff Equations (9) and (22) hold [38].

(16) $R=\left(r_{n k}\right) \in\left(c s, c s_{0}\right)$ iff Equations (9) and (23) hold with $a_{k}=0$ for all $k \in \mathbb{N}$ [38].

(17) $R=\left(r_{n k}\right) \in(c s, b v)$ iff Equation (24) holds [38].

(18) $R=\left(r_{n k}\right) \in\left(c s, b v_{0}\right)$ iff Equations (24) and (12) hold with $a_{k}=0$ for all $k \in \mathbb{N}$ [35]. 
Theorem 10. $b s(F) \supset \bigcap$ bs is valid.

Proof. Suppose $x \in b s$. If we show that $F$ is an element of $(b s, b s)$ then $x$ is element of $b s(F)$. For this, $F$ must provide Equations (8) and (9). Since $\lim _{k} f_{n k}=0$ for each $n \in \mathbb{N}$, Equation (8) is provided.

If we examine Equation (9), we find

$$
\sup _{m} \sum_{k}\left|\sum_{n=0}^{m}\left(f_{n k}-f_{n, k+1}\right)\right|=0 .
$$

Theorem 11. $b s(F) \supset \bigcap \ell_{\infty}$ is not valid.

Proof. Suppose $x=\left(x_{k}\right)=\left(f_{k+1}^{2}\right)$. Then $y=F x=(1,0,0 \ldots) \in b s$. On the other hand, $f_{k+1}^{2} \rightarrow \infty$ as $k \rightarrow \infty$. It is clear $x \in b s(F)$, but $x \notin \ell_{\infty}$. This result completes the proof.

Theorem 12. $\operatorname{cs}(F) \supset \bigcap c s$ is valid.

Proof. Suppose $x \in c s$. If we show that $F$ is element of $(c s, c s)$ then $x$ is element of $c s(F)$. For this, $F$ must provide Equations (10) and (9). Equation (9) has been provided from the Theorem 10. If we look at the Equation (10) then, for each $k \in \mathbb{N}$,

$$
\lim _{n} \sum_{k} f_{n k}=\lim _{n}\left(\frac{f_{n}}{f_{n+1}}-\frac{f_{n+1}}{f_{n}}\right)=\frac{1}{\phi}-\phi=l
$$

such that $l \in \mathbb{C}$ exists.

Theorem 13. $\operatorname{cs}(F) \supset \bigcap c$ is not valid.

Proof. Let $x=\left(x_{k}\right)=\left(f_{k+1}^{2}\right)$. Then $y=F x=(1,0,0 \ldots) \in c s$. On the other hand, $f_{k+1} \rightarrow \infty$ as $k \rightarrow \infty$. It is clear $x \in c s(F)$, but $x \notin c$. This result completes the proof.

Theorem 14. $\operatorname{cs}(F) \subset b s(F)$ is valid.

Proof. If $x \in c s(F), y=F x \in c s$. Hence, $\sum_{k} F x \in c$. Since $c \subset \ell_{\infty}, \sum_{k} F x \in l_{\infty}$. Hence, $F x \in b s$. That is, $x \in b s(F)$. This result completes the proof.

Let us take normed space $A$ and let $\left(a_{k}\right) \in A$. If there is only one scalar sequence $\left(v_{k}\right)$ such that $y=\sum_{k=0}^{\infty} v_{k} a_{k}$ and $\lim _{n \rightarrow \infty}\left\|y-\sum_{k=0}^{n} v_{k} a_{k}\right\|=0$ then $\left(a_{k}\right)$ is called a Schauder base for $A$.

Now, let us give corallary releated to Schauder basis.

Corallary 15. Let a sequence $u^{(k)}=\left\{u_{n}^{(k)}\right\}_{n \in \mathbb{N}}$ in $\operatorname{cs}(F)$ be for each $k \in \mathbb{N}$ and

$$
u_{n}^{(k)}= \begin{cases}\frac{f_{n+1}^{2}}{f_{k} f_{k+1}}, & 0 \leq k \leq n \\ 0, & n<k\end{cases}
$$

Then $\left\{u_{n}^{(k)}\right\}_{n \in \mathbb{N}}$ is a base for $\operatorname{cs}(F)$. Every $x \in \operatorname{cs}(F)$ can write as a single $x=\sum_{k=0}^{\infty} y_{k} u^{k}$ such that $y_{k}=(\hat{F} x)_{k}$ 
2.2. The Duals of $\operatorname{cs}(F)$ and $b s(F)$ and Matrix Transformations

Let us give the two lemmas to use in the next stage.

Lemma 16. Let infinite matrix $C=\left(c_{n k}\right) \frac{1}{2}$ and $a=\left(a_{n}\right) \in w$. Let us take $C=a F^{-1}$, that is,

$$
c_{n k}= \begin{cases}a_{n} f_{n k}^{-1}, & 0 \leq k \leq n \\ 0, & n<k\end{cases}
$$

for all $k, n \in \mathbb{N}, \delta \in\{c s, b s\}$. Then, $a \in\left\{\delta\left(F^{-1}\right)\right\}^{\alpha}$ iff $C \in\left\{\delta, l_{1}\right\}$.

Proof. Let $x=\left(x_{n}\right)$ and $a=\left(a_{n}\right)$ elements of $w \cdot y=\left(y_{n}\right)$ such that $y=F x$ which is defined in Equation (4). If we use to Equation (4), then

$$
a_{n} x_{n}=a_{n}\left(F^{-1} y\right)_{n}=(C y)_{n} .
$$

$a x=\left(a_{n} x_{n}\right) \in \ell_{1}$ with $x=\left(x_{n}\right) \in \mu(F)$ iff $C y \in \ell_{1}$ with $y \in \lambda$. Consequently, $C \in\left(\mu, \ell_{1}\right)$.

Lemma 17. [41] Let us take $a=\left(a_{k}\right) \in w$ and infinite matrix $C=\left(c_{n k}\right)$. Let the inverse matrix $H=\left(h_{n k}\right)$ of the triangular matrix $G=\left(g_{n k}\right)$ is given by

$$
c_{n k}= \begin{cases}\sum_{j=k}^{n} a_{j} h_{j k^{\prime}} & 0 \leq k \leq n \\ 0, & n<k\end{cases}
$$

Then, for any sequence space $\delta$,

$$
\begin{aligned}
& \delta_{G}^{\gamma}=\left\{a=\left(a_{k}\right) \in w: C \in\left(\delta, l_{\infty}\right)\right\}, \\
& \delta_{G}^{\beta}=\left\{a=\left(a_{k}\right) \in w: C \in(\delta, c)\right\} .
\end{aligned}
$$

If we consider Lemma 9, Lemma 16 and Lemma 17 together, the following is obtained;

Corallary 18. Let us take $r=\left(r_{k}\right) \in w$ and infinite matrix $A=\left(a_{n k}\right)$ and $B=\left(b_{n k}\right)$ such that

$$
a_{n k}=\left\{\begin{array}{ll}
r_{n} f_{n k}^{-1}, & 0 \leq k \leq n \\
0, & n<k
\end{array} \text { and } b_{n k}=\sum_{j=k}^{n} \frac{f_{j+1}^{2}}{f_{k} f_{k+1}} r_{j}\right.
$$

If we take $d_{1}, d_{2}, d_{3}, d_{4}, d_{5}, d_{6}, d_{7}$ and $d_{8}$ as follows:

$$
\begin{aligned}
& d_{1}=\left\{r=\left(r_{k}\right) \in w: \sup _{N, K \in \mathcal{F}}\left|\sum_{n \in \mathbb{N}} \sum_{k \in \mathbb{N}}\left(a_{n k}-a_{n, k+1}\right)\right|<\infty\right\}, \\
& d_{2}=\left\{r=\left(r_{k}\right) \in w: \sup _{N, K \in \mathcal{F}}\left|\sum_{n \in \mathbb{N}} \sum_{k \in \mathbb{N}}\left(a_{n k}-a_{n, k-1}\right)\right|<\infty\right\}, \\
& d_{3}=\left\{r=\left(r_{k}\right) \in w: \lim _{k} c_{n k}=0\right\}, \\
& d_{4}=\left\{r=\left(r_{k}\right) \in w: \exists \alpha \in \mathbb{C} \ni \lim _{n}\left(b_{n k}-b_{n, k+1}\right)=\alpha \text { for all } k \in \mathbb{N}\right\}, \\
& d_{5}=\left\{r=\left(r_{k}\right) \in w: \lim _{n} \sum_{k}\left|b_{n k}-b_{n, k+1}\right|=\sum_{k}\left|\lim \left(b_{n k}-b_{n, k+1}\right)\right|\right\}, \\
& d_{6}=\left\{r=\left(r_{k}\right) \in w: \exists \alpha \in \mathbb{C} \ni \lim _{n} b_{n k}=\alpha \text { for all } k \in \mathbb{N}\right\}, \\
& d_{7}=\left\{r=\left(r_{k}\right) \in w: \sup _{n \in \mathbb{N} k}\left|b_{n k}-b_{n, k+1}\right|<\infty\right\}, \\
& d_{8}=\left\{r=\left(r_{k}\right) \in w: \sup _{n \in \mathbb{N}}\left|\lim _{k} b_{n k}\right|<\infty\right\} .
\end{aligned}
$$


then,
(1) $\{b s(F)\}^{\alpha}=d_{1}$
(2) $\{c s(F)\}^{\alpha}=d_{2}$
(3) $\{b s(F)\}^{\beta}=d_{3} \cap d_{4} \cap d_{5}$
(4) $\{c s(F)\}^{\beta}=d_{6} \cap d_{7}$
(5) $\{b s(F)\}^{\gamma}=d_{3} \cap d_{7}$
(6) $\{c s(F)\}^{\gamma}=d_{7} \cap d_{8}$.

Theorem 19. Let $\mu \in\{c s, b s\}$ and $\lambda \subset w$. Then, $U=\left(u_{n k}\right) \in(\mu(F), \lambda)$ iff

$$
\begin{gathered}
V^{m}=\left(v_{n k}^{(m)}\right) \in(\mu, c) \text { for all } n \in \mathbb{N} \\
V=\left(v_{n k}\right) \in(\mu, \lambda)
\end{gathered}
$$

where

$$
v_{n k}^{(m)}= \begin{cases}\sum_{j=k}^{m} \frac{f_{j+1}^{2}}{f_{k} f_{k+1}} u_{n j}, & 0 \leq k \leq m \\ 0, & m<k\end{cases}
$$

and

$$
v_{n k}=\sum_{j=k}^{\infty} \frac{f_{j+1}^{2}}{f_{k} f_{k+1}} u_{n j}
$$

for all $k, m, n \in \mathbb{N}$.

Proof. Necessity part: Let us take that $A=\left(a_{n k}\right) \in(\mu(F), \lambda)$ and $x=\left(x_{k}\right) \in \mu(F)$. If we use Equation (5), then we find

$$
\begin{aligned}
\sum_{k=0}^{m} a_{n k} x_{k} & =\sum_{k=0}^{m} a_{n k} \sum_{k=0}^{m} \frac{f_{j+1}^{2}}{f_{k} f_{k+1}} y_{j} \\
& =\sum_{k=0}^{m} \sum_{j=k}^{m} \frac{f_{j+1}^{2}}{f_{k} f_{k+1}} a_{n k} y_{k}=\sum_{k=0}^{m} d_{n k}^{(m)} y_{k}=D_{n}^{(m)}(y)
\end{aligned}
$$

According to the hypothesis, for each $m \in \mathbb{N}, A_{m}(x) \in c$. Then, $V^{(m)} \in c$ for each $m \in \mathbb{N}$ and $V^{(m)} \in$ $(\mu, c) . A x=V y$ if we consider for $m \rightarrow \infty$ from Equation (30). As a result, we find $V=\left(v_{n k}\right) \in(\mu, \lambda)$.

Sufficient part: Suppose that Equations (26) and (27) are satisfied and $x=\left(x_{k}\right) \in \mu(F)$ be. By using Corollary 18 and Equations (26) and (30), we have that $y=F x \in \mu$ and

$$
V_{n}^{(m)}(y)=\sum_{k=0}^{m} v_{n k}^{(m)} y_{k}=\sum_{k=0}^{m} a_{n k} x_{k}=A_{n}^{(m)}(x) \in c .
$$

Hence, $A=\left(a_{n k}\right)_{k \in \mathbb{N}}$ exists. $A x=V y$ if we consider for $m \rightarrow \infty$ from Equation (30). Consequently, we find $A=\left(a_{n k}\right) \in(\mu(F), \lambda)$.

Theorem 20. Let $\mu \in\{b s, c s\}$ and $\lambda \subset w$ and $U=\left(u_{n k}\right)$ and $B=\left(b_{n k}\right)$ be infinite matrices. Let

$$
b_{n k}:=\frac{f_{n}}{f_{n+1}} u_{n k}-\frac{f_{n+1}}{f_{n}} u_{n-1, k} .
$$

Then, $U \in(\lambda, \mu(\hat{F}))$ iff $B \in(\lambda, \mu)$. 
Proof. Let $z=\left(z_{k}\right) \in l$ and Equation (31) exist. Then, we have

$$
\sum_{k=0}^{m} b_{n k} z_{k}=\sum_{k=0}^{m}\left(\frac{f_{n}}{f_{n+1}} a_{n k}-\frac{f_{n+1}}{f_{n}} a_{n-1, k}\right) z_{k}
$$

If we take $m \rightarrow \infty$ to Equation (32), we have that $(B z)_{\mathrm{n}}=(F(A z))_{n}$. Consequently, $A z \in \mu(F)$ iff $B z \in \mu$. That is, $B \in(\lambda, \mu)$.

Let us give almost convergent sequences space, which was first defined by Lorentz [42]. Let $t=\left(t_{k}\right) \in \ell_{\infty} . t$ is almost convergent to limit $\ell$ iff $\lim _{m \rightarrow \infty} \sum_{k=0}^{m} \frac{t_{n+k}}{m+1}=\alpha$ uniformly in $n$. It is denoted by $\hat{c}-\lim t=\alpha$. In addition, $\hat{c} s$ and $\hat{c}_{0}$ mean the spaces of almost convergent series and almost null sequences, respectively. $\hat{c}_{0}$ and $\hat{c}$ are

$$
\begin{aligned}
& \hat{c}_{0}=\left\{x=\left(x_{k}\right) \in l_{\infty}: \lim _{m \rightarrow \infty} \sum_{k=0}^{m} \frac{x_{n+k}}{m+1}=0 \text { uniformly in } n\right\}, \\
& \hat{c}=\left\{x=\left(x_{k}\right) \in l_{\infty}: \exists l \in \mathbb{C} \ni \lim _{m \rightarrow \infty} \sum_{k=0}^{m} \frac{x_{n+k}}{m+1}=\alpha \text { uniformly in } n\right\} .
\end{aligned}
$$

Now, let us take infinite matrix $R=\left(r_{n k}\right)$ and list the following:

$$
\begin{gathered}
\exists a_{k} \in \mathbb{C} \ni f-\lim r_{n k}=a_{k} \text { for each } \mathrm{k} \in \mathbb{N}, \\
\lim _{q} \sum_{k} \frac{1}{q+1}\left|\sum_{i=0}^{q} \Delta\left[\sum_{j=0}^{n+i}\left(r_{j k}-a_{k}\right)\right]\right|=0 \text { uniformly in } n, \\
\sup _{n \in \mathbb{N}} \sum_{k}\left|\Delta\left[\sum_{j=0}^{n} r_{j k}\right]\right|<\infty, \\
\exists a_{k} \in \mathbb{C} \ni f-\lim _{j=0}^{n} \sum_{j k}=a_{k} \text { for each } \mathrm{k} \in \mathbb{N}, \\
\exists a_{k} \in \mathbb{C} \ni \sum_{n} \sum_{k} r_{n k}=a_{k} \text { for each } \mathrm{k} \in \mathbb{N}, \\
\sup _{n \in \mathbb{N}} \sum_{k}\left|\sum_{j=0}^{n} r_{j k}\right|<\infty, \\
\sum_{k}|\Delta| \sum_{j=0}^{n}\left(r_{j k}-a_{k}\right] \mid=0, \\
\sup _{n \in \mathbb{N}} \sum_{k}\left|\sum_{j=0}^{n} r_{j k}\right|<\infty, q=\frac{p}{p-1}, \\
\sup _{m, n \in \mathbb{N} \mid}\left|\sum_{n=0}^{m} r_{n k}\right|<\infty, \\
\sup _{m, l \in \mathbb{N}}\left|\sum_{n=0}^{m} \sum_{k=l}^{\infty} r_{n k}\right|<\infty, \\
\sup _{m, l \in \mathbb{N}}\left|\sum_{n=0}^{m} \sum_{k=0}^{l} r_{n k}\right|<\infty,
\end{gathered}
$$




$$
\begin{aligned}
& \lim _{m} \sum_{k}\left|\sum_{n=m}^{\infty} r_{n k}\right|=0, \\
& \sum_{n} \sum_{k} r_{n k} \text { convergent }
\end{aligned}
$$

$$
\lim _{m \rightarrow \infty} \sum_{n=0}^{m}\left(r_{n k}-r_{n, k+1}\right)=a \text { for each } k \in \mathbb{N}, a \in \mathbb{C} .
$$

Lemma 21. Let infinite matrix $R=\left(r_{n k}\right)$ for all $k, n \in \mathbb{N}$. Then,

(1) $R=\left(r_{n k}\right) \in(\hat{c}, c s)$ iff Equation (23) and Equations (37)-(39) hold [43].

(2) $R=\left(r_{n k}\right) \in(c s, \hat{c})$ iff Equation (11) and Equation (33) hold [44].

(3) $R=\left(r_{n k}\right) \in(b s, \hat{c})$ iff Equations (8), (11), (33) and (34) hold [45].

(4) $R=\left(r_{n k}\right) \in(b s, \hat{c} s)$ iff Equations (8) and (34)-(36) hold [45].

(5) $R=\left(r_{n k}\right) \in(c s, \hat{c s})$ iff Equation (35) and Equation (36) hold [44].

(6) $R=\left(r_{n k}\right) \in\left(l_{\infty}, b s\right)=(c, b s)=\left(c_{0}, b s\right)$ iff Equation (37) holds [38].

(7) $R=\left(r_{n k}\right) \in\left(l_{p}, b s\right)$ iff Equation (40) holds [46].

(8) $R=\left(r_{n k}\right) \in(l, b s)$ iff Equation (41) holds [38].

(9) $R=\left(r_{n k}\right) \in(b v, b s)$ iff Equation (42) holds [38].

(10) $R=\left(r_{n k}\right) \in\left(b v_{0}, b s\right)$ iff Equation (43) holds [46].

(11) $R=\left(r_{n k}\right) \in\left(l_{\infty}, c s\right)$ iff Equation (44) holds [38].

(12) $R=\left(r_{n k}\right) \in(c, c s)$ if and only Equations (10), (37) and (45) hold [38].

(13) $R=\left(r_{n k}\right) \in\left(c s_{0}, c s\right)$ iff Equations (9) and (46) hold [38].

(14) $R=\left(r_{n k}\right) \in\left(l_{p}, c s\right)$ iff Equations (10) and (40) hold [46].

(15) $R=\left(r_{n k}\right) \in(l, c s)$ iff Equations (10) and (41) hold [46].

(16) $R=\left(r_{n k}\right) \in(b v, c s)$ if and only Equations (10), (41) and (43) hold [38].

(17) $R=\left(r_{n k}\right) \in\left(b v_{0}, c s\right)$ iffEquations (10) and (43) hold [46].

Now, suppose $v_{n k}$ and $v_{n k}^{(m)}$ which mentioned Equations (28) and (29) and give the following equations

$$
\begin{gathered}
\lim _{k} v_{n k}^{(m)}=0 \text { for each } n \in \mathbb{N}, \\
\lim _{n} \sum_{k}\left|v_{n k}^{(m)}-v_{n, k+1}^{(m)}\right|=0 \text { uniformly in } n, \\
\exists v_{k} \in \mathbb{C} \ni \lim _{n \rightarrow \infty}\left(v_{n k}^{(m)}-v_{n, k+1}^{(m)}\right)=v_{k} \text { for each } k \in \mathbb{N}, \\
\exists v_{k} \in \mathbb{C} \ni \lim _{n \rightarrow \infty} v_{n k}=0 \text { for each } n \in \mathbb{N}, \\
\exists l \in \mathbb{C} \ni \operatorname{sim}_{n \rightarrow \infty} \sum_{k}\left|v_{n k}-v_{n, k+1}\right|=l \text { uniformly in } n, \\
\lim _{m} \sum_{k}\left|\sum_{n=0} \sum_{n k}-v_{n, k+1}\right|<\infty, \\
\sup _{m \in \mathbb{N}} \sum_{k}\left|\sum_{n=0}^{m}\left(v_{n k}-v_{n, k+1}\right)\right|<\infty, \\
\lim _{m} \sum_{k}\left|\sum_{n=0}^{m}\left(v_{n k}-v_{n, k+1}\right)\right|=0, \\
\left.v_{n, k+1}\right)\left|=\sum_{k}\right| \sum_{n}\left(v_{n k}-v_{n, k+1}\right) \mid=0,
\end{gathered}
$$




$$
\begin{aligned}
& \sup _{N, K \in \mathcal{F}}\left|\sum_{n \in \mathbb{N}} \sum_{k \in \mathbb{N}}\left[\left(v_{n k}-v_{n, k+1}\right)-\left(v_{n-1, k}-v_{n-1, k+1}\right)\right]\right|<\infty, \\
& \sup _{N, K \in \mathcal{F}}\left|\sum_{n \in \mathbb{N}} \sum_{k \in \mathbb{N}}\left(v_{n k}-v_{n-1, k}\right)\right|<\infty . \\
& \exists v_{k} \in \mathbb{C} \ni \lim _{n} \mathrm{v}_{n k}^{(m)}=v_{k} \text { for each } k \in \mathbb{N} \text {, } \\
& \sup _{n} \sum_{k}\left|v_{n k}^{(m)}-v_{n, k+1}^{(m)}\right|<\infty, \\
& \sup _{n}\left|\lim _{k} v_{n k}\right|<\infty, \\
& \sup _{N, K \in \mathcal{F}}\left|\sum_{n \in \mathbb{N}} \sum_{k \in \mathbb{N}}\left[\left(v_{n k}-v_{n-1, k}\right)-\left(v_{n, k-1}-v_{n-1, k-1}\right)\right]\right|<\infty, \\
& \lim _{q} \sum_{k} \frac{1}{q+1}\left|\sum_{i=0}^{q} \Delta\left[\sum_{j=0}^{n+i}\left(v_{j k}-l_{k}\right)\right]\right|=0 \text { uniformly in } n, \\
& \sup _{n \in \mathbb{N}} \sum_{k}\left|\sum_{j=0}^{n} v_{j k}\right|<\infty, \\
& \exists l \in \mathbb{C} \ni \sum_{n} \sum_{k} v_{n k}=l \\
& \lim _{n} \sum_{k} \mid \Delta\left[\sum_{j=0}^{n}\left(v_{j k}-l_{k}\right] \mid=0\right. \\
& \sup _{n \in \mathbb{N}} \sum_{k}\left|\Delta\left[\sum_{j=0}^{n} v_{j k}\right]\right|<\infty \\
& \exists v_{k} \in \mathbb{C} \ni f-\lim \sum_{j=0}^{n} v_{j k}=v_{k} \text { for each } k \in \mathbb{N} \text {. }
\end{aligned}
$$

If we consider Theorems 19 and 20, Lemmas 9 and 21, then we give the below conclusions.

Corollary 22. Let us take $U=\left(u_{n k}\right)$ mentioned in Theorem 19. Then,

(1) $U=\left(u_{n k}\right) \in\left(b s(F), c_{0}\right)$ iff Equations (47) and (49) hold and Equation (53) holds with $l=0$.

(2) $U=\left(u_{n k}\right) \in\left(b s(F), c s_{0}\right)$ iff Equations (47)-(50) and Equation (55) hold. 
(3) $U=\left(u_{n k}\right) \in(b s(F), c)$ iff Equations (47)-(50) and Equations (52) and (53) hold.

(4) $U=\left(u_{n k}\right) \in(b s(F), c s)$ iff Equations (47)-(50) and Equation (56) hold.

(5) $U=\left(u_{n k}\right) \in\left(b s(F), l_{\infty}\right)$ iff Equations (47)-(51) hold.

(6) $U=\left(u_{n k}\right) \in(b s(F), b s)$ iff Equations (47)-(50) and Equation (54) hold.

(7) $U=\left(u_{n k}\right) \in\left(b s(F), l_{1}\right)$ iff Equations (47)-(50) and Equation (58) hold.

(8) $U=\left(u_{n k}\right) \in(b s(F), b v)$ iff Equations (47)-(50) and Equation (57) hold.

(9) $U=\left(u_{n k}\right) \in\left(b s(F), b v_{0}\right)$ iff Equations (57) and (47)-(49) and Equation (51) hold and Equation (53) also holds with $l=0$.

Corallary 23. Let us take $U=\left(u_{n k}\right)$ mentioned in Theorem 19. Then,

(1) $U=\left(u_{n k}\right) \in\left(c s(F), c_{0}\right)$ iff Equations (59), (60), Equation (51) hold and Equation (62) also holds with $v_{k}=0$ for all $k \in \mathbb{N}$.

(2) $U=\left(u_{n k}\right) \in\left(c s(F), c s_{0}\right)$ iff Equations (59), (60), Equation (54) hold and Equation (65) also holds with $v_{k}=0$ for all $k \in \mathbb{N}$.

(3) $U=\left(u_{n k}\right) \in(\operatorname{cs}(F), c)$ iff Equations (59), (60), Equation (51) and Equation (62) hold.

(4) $U=\left(u_{n k}\right) \in(c s(F), c s)$ iff Equations (59), (60), Equation (64) and Equation (65) hold.

(5) $U=\left(u_{n k}\right) \in\left(c s(F), l_{\infty}\right)$ iff Equations (51) and (59)-(61) hold.

(6) $U=\left(u_{n k}\right) \in(c s(F), b s)$ iff Equations (59), (60), (54) and (63) hold.

(7) $U=\left(u_{n k}\right) \in\left(c s(F), l_{1}\right)$ iff Equations (59), (60) and (66) hold.

(8) $U=\left(u_{n k}\right) \in(c s(F)$, bv) iff Equations (59), (60) and (68) hold.

(9) $U=\left(u_{n k}\right) \in\left(c s(F), b v_{0}\right)$ iff Equation (59), (60) and (62) hold and Equation (68) holds with $v_{k}=0$ for all $k \in \mathbb{N}$.

Corollary 24. Let us take $U=\left(u_{n k}\right)$ mentioned Theorem 19. Then,

(1) $U=\left(u_{n k}\right) \in(b s(F), \hat{c})$ iff Equations (47)-(51), (67) and (69) hold.

(2) $U=\left(u_{n k}\right) \in\left(b s(F), \hat{c}_{0}\right)$ iff Equations (47)-(51) hold and Equations (68) and (69) also hold with $v_{k}=0$ in Equation (67) and $l_{k}=0$ in Equation (69).

(3) $U=\left(u_{n k}\right) \in(c s(F), \hat{c})$ iff Equations (59), (60), (67) and (51) hold.

(4) $U=\left(u_{n k}\right) \in\left(\operatorname{cs}(F), \hat{c}_{0}\right)$ iff Equations (51), (59), (60) hold and Equation (67) also holds with $v_{k}=0$.

(5) $U=\left(u_{n k}\right) \in(b s(F), \hat{c} s)$ iff Equations (69), (73), (74) and (47)-(50), hold.

(6) $U=\left(u_{n k}\right) \in(c s(F), \hat{c s})$ iff Equations (73), (74), (59) and (60) hold.

Corallary 25. Let us take $U=\left(u_{n k}\right)$ mentioned Theorem 20. Then,

(1) $U=\left(u_{n k}\right) \in\left(l_{p}, b s(F)\right)$ iff Equation (40) holds with $b_{n k}$ instead of $r_{n k}$, where $b_{n k}$ is defined by Equation (31).

(2) $U=\left(u_{n k}\right) \in\left(l_{\infty}, b s(F)\right)=(c, b s(F))=\left(c_{0}, b s(F)\right)$ iff Equation (37) holds with $b_{n k}$ instead of $r_{n k}$, where $b_{n k}$ is defined by Equation (31).

(3) $U=\left(u_{n k}\right) \in\left(l_{1}, b s(F)\right)$ iff Equation (41) holds with $b_{n k}$ instead of $r_{n k}$, where $b_{n k}$ is defined by Equation (31).

(4) $U=\left(u_{n k}\right) \in(b v, b s(F))$ iff Equation (42) holds with $b_{n k}$ instead of $r_{n k}$, where $b_{n k}$ is defined by Equation (31).

(5) $U=\left(u_{n k}\right) \in\left(b v_{0}, b s(F)\right)$ iff Equation (43) holds $b_{n k}$ instead of $r_{n k}$, where $b_{n k}$ is defined by Equation (31).

(6) $U=\left(u_{n k}\right) \in\left(l_{\infty}, c s(F)\right)$ iff Equation (44) holds with $b_{n k}$ instead of $r_{n k}$, where $b_{n k}$ is defined by Equation (31).

(7) $U=\left(u_{n k}\right) \in(c, c s(F))$ iff (10), Equations (37) and (47) hold $b_{n k}$ instead of $r_{n k}$, where $b_{n k}$ is defined by Equation (31).

(8) $U=\left(u_{n k}\right) \in\left(c s_{0}, c s(F)\right)$ iff Equations (9) and (46) hold with $b_{n k}$ instead of $r_{n k}$, where $b_{n k}$ is defined by Equation (31).

(9) $U=\left(u_{n k}\right) \in\left(l_{p}, c s(F)\right)$ iff Equations (10) and (40) hold with $b_{n k}$ instead of $r_{n k}$, where $b_{n k}$ is defined by Equation (31). 
(10) $U=\left(u_{n k}\right) \in(l, c s(F))$ iff Equations (10) and (41) hold with $b_{n k}$ instead of $r_{n k}$, where $b_{n k}$ is defined by Equation (31).

(11) $U=\left(u_{n k}\right) \in(b v, c s(F))$ iff Equations (10), (41) and (43) hold with $b_{n k}$ instead of $r_{n k}$, where $b_{n k}$ is defined by Equation (31).

(12) $U=\left(u_{n k}\right) \in\left(b v_{0}, c s(F)\right)$ iff Equations (10) and (43) hold $b_{n k}$ instead of $r_{n k}$, where $b_{n k}$ is defined by Equation (31).

(13) $U=\left(u_{n k}\right) \in(\hat{c}, c s(F))$ iff Equation (65) and Equations (70)-(72) hold with $b_{n k}$ instead of $v_{n k}$, where $b_{n k}$ is defined by Equation (31).

\section{Discussion}

Kızmaz [47] first introduced the difference sequence operator in 1981. Generalized difference sequence spaces were characterized and investigated by Kiriş̧̧i and Başar [4] in 2010. Kara [27] first defined the Fibonacci Difference Matrix $F$, which created the Fibonacci sequence $\left(f_{n}\right)$ in 2013 . He also introduced the new sequence spaces $\ell_{p}(F)$ and $\ell_{\infty}(F)$; where $1 \leq p<\infty$. The spaces $c(F(r, s))$ and $c_{0}(F(r, s))$ were introduced by Candan [28] in 2015. In 2015, the sequence space $\ell_{p}(F(r, s))$ was introduced and studied by Candan and Kara [19]; where $1 \leq p \leq \infty$. In addtion, a class of compact operators on $\ell_{p}(F)$ and $\ell_{\infty}(F)$ was characterized by Kara et al. [32], where $1 \leq p<\infty$.

In the present study, we introduced the domain of a triangular infinite matrix on a sequences space. We described spaces $c s(F)$ and $b s(F)$, where $F, c s$, and $b s$ are the Fibonacci Difference Matrix, convergent and bounded series, respectively. It was demonstrated that $b s(F)$ are the linear spaces, and given that $\operatorname{cs}(F)$ is linear space in Theorem 6. without proof and, they have the same norm

$$
\|x\|=\sup _{n \in \mathbb{N}}\left|\sum_{j=0}^{n}\left(\frac{f_{k}}{f_{k+1}} x_{k}-\frac{f_{k+1}}{f_{k}} x_{k-1}\right)\right|
$$

where $x \in c s(F)$ or $x \in b s(F)$. It was found that they are Banach spaces. In addition, inclusions theorems were examined and found. Finally, the $\gamma, \beta, \alpha$-duals of them were calculated. Finally, some matrix transformations as a main result were given.

Author Contributions: This article was written with equal contributions from both authors. The final manuscript was read and approved by both authors.

Funding: This research received no external funding.

Acknowledgments: We thank the responsible editors and reviewers for their contribution for the improvement of this paper. We also thank Mehmet Kanoğlu for his help in reviewing English of the paper.

Conflicts of Interest: The authors declare no conflict of interest.

\section{References}

1. Cooke, R.G. Infinite Matrices and Sequence Spaces; Macmillan and Co. Limited: London, UK, 1950.

2. Ng, P.-N.; Lee, P.-Y. Cesaro sequence space of non-absolute type. Comment. Math. Prace Mat. 1978, 20, 429-433.

3. Wang, C.S. On Nörlund Sequence Space. Tamkang J. Math. 1978, 9, 269-274.

4. Malkovsky, E. Recent results in the theory of matrix transformations in sequence spaces. Mat. Vesnik 1997, 49, 187-196.

5. Altay, B.; Başar, F. On some Euler sequence spaces of nonabsolute type. Ukranian Math. J. 2005, 57, 3-17. [CrossRef]

6. Malkowsky, E.; Savaş, E. Matrix transformations between sequence spaces of generalized weightedmean. Appl. Math. Comput. 2004, 147, 333-345.

7. Aydın, C.; Başar, F. On the new sequence spaces which include the spaces $c_{0}$ and c. Hokkaido Math. J. 2004, 33, 1-16. [CrossRef] 
8. Kirişçi, M.; Başar, F. Some new sequence spaces derived by the domain of generalized difference matrix. Comput. Math. Appl. 2010, 60, 1299-1309. [CrossRef]

9. Şengönül, M.; Başar, F. Some new Cesàro sequence spaces of non-absolute type which include thespaces $c_{0}$ and c. Soochow J. Math. 2005, 31, 107-119.

10. Altay, B.; Başar, F. Some paranormed Riezs sequence spaces of non-absolute type. Southeast Asian Bull. Math. 2006, 30, 591-608.

11. Mursaleen, M.; Noman, A.K. On the spaces of $\lambda$-convergent and bounded sequences. Thai J. Math. 2010, 8, 311-329.

12. Candan, M. Domain of the double sequential band matrix in the classical sequence spaces. J. Inequal. Appl. 2012, 2012, 281. [CrossRef]

13. Candan, M.; Kayaduman, K. Almost convergent sequence space derived by generalized Fibonacci matrix and Fibonacci core. Br. J. Math. Comput. Sci. 2015, 7, 150-167. [CrossRef]

14. Candan, M. Almost convergence and double sequential band matrix. Acta Math. Sci. 2014, 34, $354-366$. [CrossRef]

15. Candan, M. A new sequence space isomorphic to the space $\ell_{p}$ and compact operators. J. Math. Comput. Sci. 2014, 4, 306-334.

16. Candan, M. Domain of the double sequential band matrix in the spaces of convergent and null sequences. Adv. Differ. Equ. 2014, 2014, 163. [CrossRef]

17. Candan, M.; Güneş, A. Paranormed sequence space of non-absolute type founded using generalized difference matrix. Proc. Nat. Acad. Sci. India Sect. A 2015, 85, 269-276. [CrossRef]

18. Candan, M. Some new sequence spaces derived from the spaces of bounded, convergent and null sequences. Int. J. Mod. Math. Sci. 2014, 12, 74-87.

19. Candan, M.; Kara, E.E. A study on topological and geometrical characteristics of new Banach sequence spaces. Gulf J. Math. 2015, 3, 67-84.

20. Candan, M.; Kılınç, G. A different look for paranormed Riesz sequence space derived by Fibonacci matrix. Konuralp J. Math. 2015, 3, 62-76.

21. Şengönül, M.; Kayaduman, K. On the Riesz almost convergent sequence space. Abstr. Appl. Anal. 2012, 2012, 691694. [CrossRef]

22. Kayaduman, K.; Şengönül, M. The spaces of Cesàro almost convergent sequences and core theorems. Acta Math. Sci. 2012, 32B, 2265-2278. [CrossRef]

23. Çakan, C.; Çoşkun, H. Some new inequalities related to the invariant means and uniformly bounded function sequences. Appl. Math. Lett. 2007, 20, 605-609. [CrossRef]

24. Çoşkun, H.; Çakan, C. A class of statistical and $\sigma$-conservative matrices. Czechoslovak Math. J. 2005, 55, 791-801. [CrossRef]

25. Çoşkun, H.; Çakan, C.; Mursaleen. On the statistical and $\sigma$-cores. Studia Math. 2003, 154, $29-35$.

26. Kayaduman, K.; Furkan, H. Infinite matrices and $\sigma^{(\mathrm{A})}$-core. Demonstratio Math. 2006, 39, 531-538. [CrossRef]

27. Kara, E.E. Some topological and geometrical properties of new Banach sequence spaces. J. Inequal. Appl. 2013, 2013, 38. [CrossRef]

28. Candan, M. A new approach on the spaces of generalized Fibonacci difference null and convergent sequences. Math. Aeterna 2015, 5, 191-210.

29. Yaşar, F.; Kayaduman, K. A Different Study on the Spaces of Generalized Fibonacci Difference bs and cs Spaces Sequence. Symmetry 2018, 10, 274. [CrossRef]

30. Kayaduman, K.; Yaşar, F. On Domain of Nörlund Matrix. Mathematics 2018, 6, 268. [CrossRef]

31. Koshy, T. Fibonacci and Lucas Numbers with Applications; Wiley: New York, NY, USA, 2001.

32. Kara, E.E.; Başarır, M.; Mursaleen, M. Compact operators on the Fibonacci difference sequence spaces $\ell_{p}(\hat{F})$ and $\ell_{\infty}(F)$. In Proceedings of the 1st International Eurasian Conference on Mathematical Sciences and Applications, Prishtine, Kosovo, 3-7 September 2012.

33. Başarır, M.; Başar, F.; Kara, E.E. On the spaces of Fibonacci difference null convergent sequences. arXiv 2013, arXiv:1309.0150v1.

34. Demiriz, S.; Kara, E.E.; Başarır, M. On the Fibonacci almost convergent sequence spaces and Fibonacci core. Kyungpook Math. J. 2015, 55, 355-372. [CrossRef]

35. Stieglitz, M.; Tietz, H. Matrix transformationen von folgenräumen eine ergebnisübersicht. Math. Z. 1997, 154, 1-16. [CrossRef] 
36. Wilansky, A. Summability through Functional Analysis, North-Holland Mathematics Studies; Elsevier: Amsterdam, The Netherlands, 1984; Volume 85.

37. Grosse-Erdman, K.-G. Matrix transformations between the sequence space of Maddox. J. Math Anal. Appl. 1993, 180, 223-238. [CrossRef]

38. Zeller, K. Allgemeine Eigenschaften von Limitierungsverfahren die auf Matrixtransformationen beruhen; Wissenschaftliche Abhandlung: Berlin, Germany, 1949.

39. Hill, J.D. On the Space $(\gamma)$ of Convergent Series, Tohoku Mathematical Journal, First Series, 1939. Available online: https://www.jstage.jst.go.jp/article/tmj1911/45/0/45_0_332/_article/_char/ja/ (accessed on 19 March 2010).

40. Dienes, P. Taylor Series. An Introduction to the Theory of Functions of a Complex Veriable; Clarendon Press: Oxford, UK; New York, NY, USA, 1957.

41. Altay, B.; Başar, F. Certain topological properties and duals of the matrix domain of a triangle matrix in a sequence space. J. Math. Anal. Appl. 2007, 336, 632-645. [CrossRef]

42. Lorentz, G.G. A contribution to the theory of divergent sequences. Acta Math. 1948, 80, 167-190. [CrossRef]

43. Başar, F. Strongly-conservative sequence-to-series matrix transformations. Erc. Üni. Fen Bil. Derg. 1989, 5, 888-893.

44. Başar, F.; Çolak, R. Almost-conservative matrix transformations. Turkish J. Math. 1989, 13, 91-100.

45. Başar, F.; Solak, İ. Almost-coercive matrix transformations. Rend. Mat. Appl. 1991, 11, 249-256.

46. Jakimovski, A.; Russell, D.C. Matrix mapping between BK-spaces. Bull. Lond. Math. Soc. 1972, 4, 345-353. [CrossRef]

47. Kızmaz, H. On certain sequence spaces. Can. Math. Bull. 1981, 24, 169-176. [CrossRef]

(C) 2019 by the authors. Licensee MDPI, Basel, Switzerland. This article is an open access article distributed under the terms and conditions of the Creative Commons Attribution (CC BY) license (http:/ / creativecommons.org/licenses/by/4.0/). 